\title{
Reciprocal patterns of c-Fos expression in the medial prefrontal cortex and amygdala after extinction and renewal of conditioned fear
}

\author{
Ewelina Knapska ${ }^{1,3}$ and Stephen Maren ${ }^{2,4}$ \\ ${ }^{1}$ Department of Psychology, University of Michigan, Ann Arbor, Michigan 48109, USA; ${ }^{2}$ Neuroscience Program, \\ University of Michigan, Ann Arbor, Michigan 48109, USA
}

\begin{abstract}
After extinction of conditioned fear, memory for the conditioning and extinction experiences becomes context dependent. Fear is suppressed in the extinction context, but renews in other contexts. This study characterizes the neural circuitry underlying the context-dependent retrieval of extinguished fear memories using c-Fos immunohistochemistry. After fear conditioning and extinction to an auditory conditioned stimulus (CS), rats were presented with the extinguished CS in either the extinction context or a second context, and then sacrificed. Presentation of the CS in the extinction context yielded low levels of conditioned freezing and induced c-Fos expression in the infralimbic division of the medial prefrontal cortex, the intercalated nuclei of the amygdala, and the dentate gyrus (DG). In contrast, presentation of the CS outside of the extinction context yielded high levels of conditioned freezing and induced c-Fos expression in the prelimbic division of the medial prefrontal cortex, the lateral and basolateral nuclei of the amygdala, and the medial division of the central nucleus of the amygdala. Hippocampal areas CA1 and CA3 exhibited c-Fos expression when the CS was presented in either context. These data suggest that the context specificity of extinction is mediated by prefrontal modulation of amygdala activity, and that the hippocampus has a fundamental role in contextual memory retrieval.
\end{abstract}

Considerable interest has emerged in recent years in the neural mechanisms underlying the associative extinction of learned fear (Maren and Quirk 2004; Myers et al. 2006; Quirk and Mueller 2008). Notably, extinction is a useful model for important aspects of exposure-based therapies for the treatment of human anxiety disorders such as panic disorder and post-traumatic stress disorder (PTSD) (Bouton et al. 2001, 2006). During extinction, a conditioned stimulus (CS) is repeatedly presented in the absence of the unconditioned stimulus (US), a procedure that greatly reduces the magnitude and probability of the conditioned response (CR). After the extinction of fear, there is substantial evidence that extinction does not erase the original fear memory, but results in a transient inhibition of fear. For example, extinguished fear responses return after the mere passage of time (i.e., spontaneous recovery) or after a change in context (i.e., renewal) (Bouton et al. 2006; Ji and Maren 2007). In other words, extinguished fear is context specific. The return of fear after extinction is a considerable challenge for maintaining long-lasting fear suppression after exposure-based therapies (Rodriguez et al. 1999; Hermans et al. 2006; Effting and Kindt 2007; Quirk and Mueller 2008).

In the last several years, considerable progress has been made in understanding the neural mechanisms underlying the context specificity of fear extinction. For example, lesions or inactivation of the hippocampus prevent the renewal of fear when an extinguished CS is presented outside of the extinction context (Corcoran and Maren 2001, 2004; Corcoran et al. 2005; Ji and Maren 2005, 2008; Hobin et al. 2006). In addition, neurons in the basolateral complex of the amygdala exhibit context-specific spike firing to extinguished CSs (Hobin et al. 2003; Herry et al. 2008),

\footnotetext{
${ }^{3}$ Present address: Nencki Institute of Experimental Biology, 02-093 Warsaw, Poland. ${ }^{4}$ Corresponding author.

E-mail maren@umich.edu; fax (734) 763-7480.
}

Article is online at http://www.learnmem.org/cgi/doi/10.1101//m.1463909. and this requires hippocampal input (Maren and Hobin 2007). Indeed, amygdala neurons that fire more to extinguished CSs outside of the extinction context are monosynaptically excited by hippocampal stimulation (Herry et al. 2008). In contrast, neurons that responded preferentially to extinguished CSs in the extinction context receive synaptic input from the medial prefrontal cortex (Herry et al. 2008).

The prevalent theory of the interactions between the prefrontal cortex, hippocampus, and amygdala that lead to regulation of fear by context assumes that when animals experience an extinguished CS in the extinction context, the hippocampus drives prefrontal cortex inhibition of the amygdala to suppress fear (Hobin et al. 2003; Maren and Quirk 2004; Maren 2005). When animals encounter an extinguished CS outside of the extinction context, the hippocampus is posited to inhibit the prefrontal cortex and thereby promote amygdala activity required to renew fear. The hippocampus may also drive fear renewal through its direct projections to the basolateral amygdala (Herry et al. 2008). Although this model accounts for much of the extant literature on the context specificity of extinction, it is not known whether the nodes of this hypothesized neural network are coactive during the retrieval of fear and extinction memories. As a first step in addressing this issue, we used ex vivo c-Fos immunohistochemistry (e.g., Knapska et al. 2007) to generate a functional map of the neural circuits involved in the contextual retrieval of fear memory after extinction. Our results reveal reciprocal activity in prefrontalamygdala circuits involved in extinction and renewal and implicate the hippocampus in hierarchical control of contextual memory retrieval within these circuits.

\section{Results}

Behavior

Rats were conditioned and extinguished as previously described (Corcoran et al. 2005). Twenty-four hours after extinction, the rats 
were given a brief retrieval test consisting of two CS exposures to activate neural circuits associated with the retrieval of extinction or fear memories. Conditional freezing was averaged across the retrieval test (including both the CS and post-CS periods) and is shown in Figure 1 . As we and others have previously shown, conditional freezing was low when the extinguished CS was presented in the extinction context (EXT-SAME), but renewed to levels no different from nonextinguished controls (NO-EXT) when the CS was presented outside of the extinction context (EXT-DIFF). Rats that were not conditioned (NO-COND) did not exhibit conditional freezing. These impressions were confirmed in a one-way ANOVA that revealed a significant main effect of group $\left(F_{(3,27)}=7.3, P<0.001\right)$. Post-hoc Fisher's protected leastsignificant difference (PLSD) tests $(P<0.05)$ confirmed that there were significant differences between the EXT-DIFF and EXT-SAME groups, the EXT-DIFF and NO-COND groups, and the NO-EXT and NO-COND groups.

\section{c-Fos expression}

The level of c-Fos expression in the brain was enhanced by both the renewal of fear outside of the extinction context (EXT-DIFF group) and successful inhibition of fear inside the extinction context (EXT-SAME group) relative to animals that were either not conditioned (NO-COND group) or conditioned, but not extinguished (NO-EXT group). However, the pattern of c-Fos expression within various brain structures differed dramatically among these behavioral conditions (see Table 1 for all brain structures). We focused our analysis on the medial prefrontal cortex, amygdala, and hippocampus, insofar as these structures have been implicated in the renewal and extinction of fear. Representative coronal sections from these brain areas are shown in Figure 2.

Quantification of c-Fos immunopositive nuclei revealed increased c-Fos expression in the infralimbic division of the prefrontal cortex (IL) when animals were presented with an extinguished CS (Fig. 3). Interestingly, c-Fos expression was the highest in IL when the CS was presented in the extinction context (EXTSAME), a condition in which animals suppress their fear to the extinguished CS. In contrast, c-Fos activation in the prelimbic division of the prefrontal cortex (PRL) was only elevated in rats that renewed their fear to the CS outside of the extinction context (EXT-DIFF; Fig. 3). In both cases, changes in Fos expression depended on the extinction history of the CS insofar as presentation of a nonextinguished CS did not evoke a significant increase in Fos expression (NO-EXT) relative to rats that were not conditioned (NO-COND).

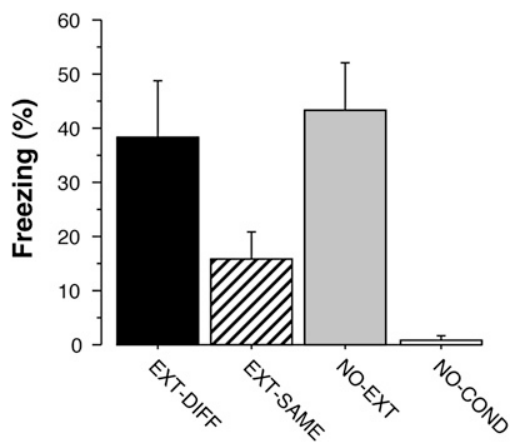

Figure 1. Mean ( \pm SEM) percentage of freezing during the test session. Rats that were tested in the context different from the context in which fear response was extinguished (EXT-DIFF) showed a renewal of fear. They froze at similar levels to animals in the NO-EXT group, and animals in both of the EXT-DIFF and NO-EXT groups exhibited greater levels of freezing than rats in the EXT-SAME and NO-COND groups.
This reciprocal pattern of prefrontal cortical Fos expression was paralleled by similar changes in the brain regions to which the medial prefrontal cortex is connected. In the amygdala, for example, we observed that the renewal of fear was associated with increased c-Fos expression in the dorsolateral part of the lateral nucleus (LAdl), the basolateral nucleus (BL), and the medial division of the central nucleus (CEm) relative to rats expressing extinction (Fig. 4). We also observed similar changes in the dorsal raphe nuclei (DRN) and the nucleus accumbens shell (NAcc shell; Table 1). In contrast to the renewal of fear, the expression of extinction was found to selectively increase c-Fos expression in the intercalated nuclei (ITC) of the amygdala (Fig. 4), an effect that was similar to that observed in IL.

Within the hippocampus, we observed that extinguished CSs elevated c-Fos expression and, like the central nucleus (CEI) and bed nucleus of the stria terminalis (BST), the degree of c-Fos expression was similar in the renewal and extinction conditions. This effect was most robust in area CA1, which exhibited increases in both the dorsal and ventral hippocampus in the extinction and renewal conditions (Fig. 5). Hippocampal area CA3 also exhibited increases in c-Fos expression in the renewal and extinction conditions, particularly in the dorsal hippocampus. In addition to the hippocampus, the CEl and BST exhibited similar increases in c-Fos expression in both the renewal and extinction conditions (Table 1). Unlike the hippocampus, c-Fos expression in the dentate gyrus (DG) was selectively increased in the extinction group, particularly in the ventral dentate gyrus (Fig. 5). This selective increase in c-Fos expression among rats expressing extinction paralleled the expression patterns in the IL and ITC.

The majority of brain regions we sampled exhibited similar levels of c-Fos expression in the NO-EXT and NO-COND conditions. This suggests that the expression of fear to an acoustic CS was not itself sufficient to induce c-Fos. However, there were two brain areas in which the mere expression of fear appeared to be associated with c-Fos expression: LAdl (Fig. 4) and PAGv (Table 1). Furthermore, there was a trend toward an increase in c-Fos expression in the NO-EXT group in the BST. Hence, c-Fos expression in LAdl and PAGv was associated with the expression of conditional freezing, whereas activity in the PRL, BL, and CEm was associated with only the renewal of fear.

To further explore the relationship of c-Fos expression to the expression of conditional fear after extinction, we examined the correlation between c-Fos expression and conditional freezing in brain structures involved in fear renewal and extinction. We observed significant correlations among rats in the EXT-DIFF and EXT-SAME groups between c-Fos expression and conditional freezing in CEm $(r=0.67, P<0.01$; Fig. 6 , top left), DGd $(r=-0.57$; $P<0.05)$, and DRN $(r=0.62, P<0.05)$. The particularly strong correlation between freezing and c-Fos expression in CEm is interesting in light of anatomical models that have suggested the expression of fear after extinction is regulated by prefrontal cortical modulation of CEm activity via the intercalated nuclei (Quirk et al. 2003). We were, therefore, interested in identifying brain structures with c-Fos expression levels that correlated with those in CEm. As shown in Figure 6, several brain regions exhibited significant correlations with CEm c-Fos expression. Importantly, c-Fos expression in the infralimbic cortex, intercalated nuclei, and ventral CA1 were negatively correlated with c-Fos expression in CEm. Conversely, c-Fos expression in the basolateral nucleus of the amygdala and the dorsal raphe nuclei were positively correlated with c-Fos expression in CEm. This suggests a large network of brain areas that interact with the CEm to regulate the expression of conditional freezing after extinction. Of course, correlations alone do not reveal the nature of the functional interactions between these brain structures - they may be either direct, indirect, or due to activity in a common input. Nonetheless, they 
Table 1. Renewal of fear and c-Fos expression in different brain structures

\begin{tabular}{|c|c|c|c|c|c|c|}
\hline Brain region & $F$ & $P$ & EXT-DIFF & EXT-SAME & NO-EXT & NO-COND \\
\hline$\overline{P R L}$ & 5.23 & $<0.01$ & 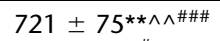 & $449 \pm 43$ & $448 \pm 79$ & $373 \pm 56$ \\
\hline IL & 9.95 & $<0.001$ & $453 \pm 81^{\wedge \#}$ & $721 \pm 97^{* \wedge \wedge \wedge \# \# \#}$ & $222 \pm 47$ & $241 \pm 66$ \\
\hline NAcc core & 1.92 & $\mathrm{~ns}$ & $180 \pm 19$ & $132 \pm 26$ & $124 \pm 11$ & $137 \pm 14$ \\
\hline NAcc shell & 2.88 & $<0.05$ & $280 \pm 23^{\wedge \#}$ & $210 \pm 38$ & $190 \pm 17$ & $190 \pm 18$ \\
\hline ACC & 1.23 & ns & $255 \pm 24$ & $192 \pm 34$ & $223 \pm 18$ & $199 \pm 24$ \\
\hline $\mathrm{BL}$ & 5.96 & $<0.01$ & $289 \pm 24^{\wedge \wedge \wedge} \wedge^{\prime}$ & $243 \pm 17^{\wedge}$ & $174 \pm 17$ & $228 \pm 19$ \\
\hline CEI & 9.20 & $<0.001$ & $347 \pm 30^{\wedge \wedge \wedge \# \# \# ~}$ & $300 \pm 22^{\wedge \wedge \# \#}$ & $206 \pm 22$ & $201 \pm 20$ \\
\hline CEm & 6.03 & $<0.01$ & 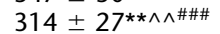 & $209 \pm 21$ & $219 \pm 17$ & $200 \pm 18$ \\
\hline LAdl & 6.29 & $<0.01$ & $130 \pm 9^{* * \# \#}$ & $69 \pm 15$ & $116 \pm 14^{\$ \&}$ & $70 \pm 10$ \\
\hline LAvl & 2.00 & $\mathrm{~ns}$ & $114 \pm 13$ & $70 \pm 14$ & $88 \pm 15$ & $75 \pm 12$ \\
\hline $\mathrm{BM}$ & 0.30 & ns & $77 \pm 8$ & $88 \pm 16$ & $73 \pm 16$ & $73 \pm 8$ \\
\hline MEpd & 0.02 & ns & $92 \pm 7$ & $91 \pm 9$ & $92 \pm 10$ & $94 \pm 11$ \\
\hline MEpv & 0.20 & ns & $92 \pm 10$ & $107 \pm 24$ & $101 \pm 13$ & $91 \pm 19$ \\
\hline BST & 2.75 & $=0.06$ & $177 \pm 23^{\#}$ & $190 \pm 32^{\#}$ & $169 \pm 17$ & $113 \pm 6$ \\
\hline ITC & 8.78 & $<0.001$ & $117 \pm 19$ & $198 \pm 14^{\star * \wedge \wedge \wedge \# \# \# ~}$ & $87 \pm 14$ & $112 \pm 16$ \\
\hline CA1d & 5.43 & $<0.01$ & $673 \pm 54^{\wedge \#}$ & $716 \pm 60^{\wedge \wedge \# \#}$ & $522 \pm 36$ & $506 \pm 37$ \\
\hline CA3d & 6.25 & $<0.01$ & $575 \pm 35^{\wedge \# \#}$ & $607 \pm 46^{\wedge \wedge \# \#}$ & $445 \pm 42$ & $424 \pm 21$ \\
\hline DGd & 2.61 & ns & $447 \pm 42$ & $568 \pm 52$ & $453 \pm 26$ & $368 \pm 52$ \\
\hline CA1v & 7.01 & $<0.01$ & $690 \pm 50^{\wedge}$ & $853 \pm 103^{\wedge \wedge \wedge \# \# ~}$ & $462 \pm 23$ & $590 \pm 55$ \\
\hline CA3v & 1.88 & ns & $431 \pm 37$ & $510 \pm 54$ & $363 \pm 23$ & $435 \pm 62$ \\
\hline DGv & 9.06 & $<0.001$ & $493 \pm 37$ & $677 \pm 36^{* * \wedge \wedge \wedge \# \# \# ~}$ & $489 \pm 40$ & $425 \pm 28$ \\
\hline SUBv & 2.41 & ns & $541 \pm 52$ & $643 \pm 72$ & $402 \pm 51$ & $572 \pm 81$ \\
\hline MGd & 1.21 & ns & $94 \pm 7$ & $112 \pm 7$ & $104 \pm 5$ & $101 \pm 9$ \\
\hline MGv & 0.59 & ns & $88 \pm 8$ & $103 \pm 9$ & $100 \pm 10$ & $96 \pm 8$ \\
\hline PAGd & 2.25 & ns & $132 \pm 14$ & $125 \pm 12$ & $124 \pm 9$ & $95 \pm 10$ \\
\hline PAGI & 1.26 & ns & $146 \pm 17$ & $140 \pm 17$ & $146 \pm 18$ & $110 \pm 10$ \\
\hline PAGV & 6.98 & $<0.01$ & $145 \pm 12^{* \# \# \#}$ & $116 \pm 7$ & $133 \pm 6^{\# \$ \$}$ & $89 \pm 11$ \\
\hline DRN & 5.08 & $<0.01$ & $183 \pm 24^{\star * \star \wedge \# \#}$ & $103 \pm 6$ & $137 \pm 9$ & $124 \pm 14$ \\
\hline
\end{tabular}

Results of one-way ANOVAs and post-hoc Fisher's PLSD tests for each brain region. c-Fos levels are shown as numbers of immunopositive cell nuclei per area of each structure expressed in millimeters squared ( \pm SEM). Significance levels (Fisher's PLSD tests) refer to differences between EXT-SAME and EXTDIFF $\left({ }^{*} P<0.05,{ }^{* *} P<0.01,{ }^{* * *} P<0.001\right)$; between NO-EXT and EXT-SAME or EXT-DIFF $(\wedge p<0.05, \wedge \wedge p<0.01, \wedge \wedge \wedge p<0.001)$; between NO-COND and EXT-SAME or EXT-DIFF $\left({ }^{\#} P<0.05,{ }^{\# \#} P<0.01,{ }^{\# \# \# P<0.001)}\right.$; between NO-EXT and NO-COND $\left({ }^{\$} P<0.05, \${ }^{\$} P<0.01\right)$, as well as between NO-EXT and EXT-SAME ( $\left.{ }^{\infty} P<0.05\right)$ groups; (ns) no significant difference between groups.

provide converging evidence for a neural network that is involved in the contextual retrieval of fear memory after extinction.

\section{Discussion}

It has been previously demonstrated that the acquisition of an extinction memory is associated with c-Fos expression in prefrontalamygdala circuits (e.g., Herry and Mons 2004; Santini et al. 2004; Hefner et al. 2008; Muigg et al. 2008). We now demonstrate that distinct neural circuits are engaged by the retrieval of fear memories after extinction, and the nature of these circuits depends upon the context in which memory retrieval occurs. Rats that were presented with an extinguished CS outside of the extinction context renewed their fear to the CS and exhibited elevated c-Fos expression in the PRL, LAdl, BL, CEm, NAcc shell, and PAGv. On the other hand, rats that suppressed their fear to an extinguished $\mathrm{CS}$ in the extinction context exhibited elevated c-Fos expression in the IL, ITC, and DG. There was also a group of structures, including CEl, BST, and hippocampal areas CA1 and CA3, that were active in both behavioral conditions. Hence, this study has revealed reciprocal neural networks that are involved in the contextual retrieval of fear memory after extinction.

The expression of c-Fos in the medial prefrontal cortex during the retrieval of extinction memories in the present study is consistent with a large body of work implicating this region in acquiring extinction memories. For example, Quirk and colleagues have found that IL lesions interfere with retrieval of fear extinction (Quirk et al. 2000; Lebrón et al. 2004). It has also been shown that pairing CSs with electrical stimulation of the IL accelerates extinction and reduces conditioned freezing (Milad and Quirk 2002; Milad et al. 2004). Moreover, medial prefrontal c-Fos expression is elevated immediately after extinction training (Herry and Mons 2004; Santini et al. 2004) and Hefner et al. (2008) have shown that impairments in extinction in the 129S1 mouse strain are accompanied by reduced c-Fos and Zif268 expression in the IL shortly after extinction training. According to Quirk et al. (2003), the IL sends projections to inhibitory neurons in the ITC, which in turn inhibit CEm. By this view, fear suppression after extinction is mediated by prefrontal cortical inhibition of amygdala output. Consistent with these results are observations that chemical stimulation of the IL increases c-Fos labeling in the ITC (Berretta et al. 2005), and lesions of the ITC impair the expression of extinction (Likhtik et al. 2008). Our data further extend this model by demonstrating that the expression of extinction is associated with parallel increases in c-Fos expression in both the IL and ITC that correlate with CEm c-Fos expression.

The renewal of fear outside of the extinction context was associated with a different pattern of c-Fos expression in corticoamygdaloid circuits. Specifically, we observed high c-Fos expression in the CEm as well as in the lateral and basal nuclei of the amygdala that was accompanied by elevated c-Fos expression in the PRL. These results are consistent with the emerging view that PRL is involved in the expression of conditioned fear (Akirav et al. 2006; Sierra-Mercado et al. 2006; Vidal-Gonzalez et al. 2006; Corcoran and Quirk 2007). Moreover, opposite roles for the PRL and IL in fear expressions are supported by recent work showing that neurons in the PRL and IL respond in the opposite manner to conditioned tones (Gilmartin and McEchron 2005). Considering the anatomical connections of the medial prefrontal cortex (Gabbott et al. 2003; Vertes 2004), it is possible that the PRL drives the renewal of fear through its extensive anatomical projections to the basolateral nucleus of the amygdala. Indeed, c-Fos expression 

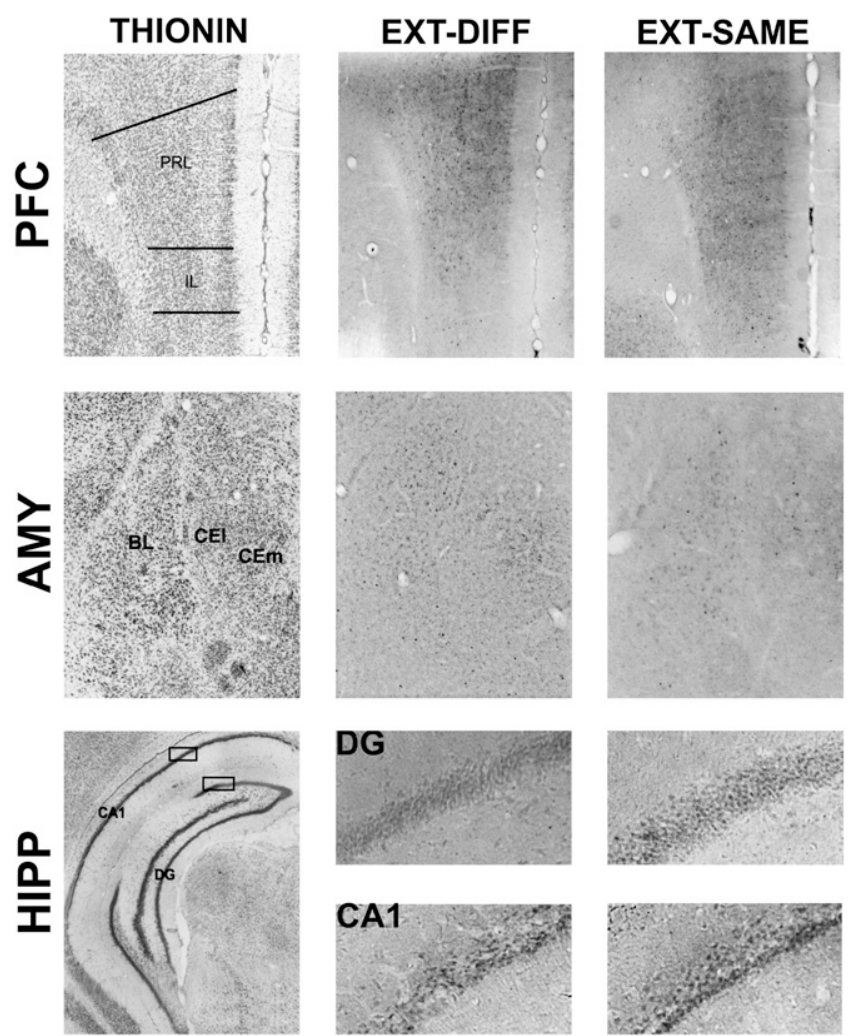

Figure 2. Adjacent sections indicating neuronal nuclei (thionin stain; left) and c-Fos immunoreactivity (black dots) observed in the EXT-DIFF (middle), and EXT-SAME (right) groups in the medial prefrontal cortex (PFC), the amygdala (AMY), and the hippocampus (HIPP).

in the basolateral nucleus was positively correlated with CEm c-Fos expression, which in turn was strongly related to levels of conditional freezing during the renewal of fear.

An alternative possibility is that the hippocampus regulates the renewal of fear (Maren and Quirk 2004; Ji and Maren 2007). We have previously reported that lesions or inactivation of the hippocampus prevents both the behavioral renewal of fear (Corcoran and Maren 2001, 2004; Corcoran et al. 2005; Ji and Maren 2005, 2008; Hobin et al. 2006) as well as "neuronal renewal" (i.e., the increase in CS-elicited spike firing to a CS presented outside of the extinction context) (Hobin et al. 2003; Maren and Hobin 2007). Moreover, single units in the basolateral nucleus of the amygdala that preferentially fire to CSs outside of the extinction context receive synaptic input from the hippocampus (Herry et al. 2008). Consistent with this model, we find that c-Fos expression in both the basolateral amygdala and hippocampal areas CA1 and CA3 is elevated in rats, renewing fear outside of the extinction context.

Interestingly, however, the elevation of hippocampal c-Fos expression was not limited to rats in the renewal condition. Indeed, the expression of extinction also elevated c-Fos expression in hippocampal areas CA1 and CA3, and c-Fos expression in the DG was selectively increased in the extinction group. These data suggest that hippocampal pyramidal cells are engaged in processing extinguished CSs, independent of where they are presented. As such, they may be involved in regulating not only the renewal of fear, but also the expression of extinction. Consistent with this possibility, we have found that hippocampal inactivation impairs extinction under some conditions (Corcoran et al. 2005). Hence, the hippocampus may have a general role in contextual process- ing that is involved in regulating both fear extinction and renewal. This is consistent with a large literature revealing an essential role for the hippocampus in contextual memory (Sanders et al. 2003; Matus-Amat et al. 2004; Rudy and Matus-Amat 2005; Wiltgen et al. 2006). Contextual memory retrieval may also involve BST and CEl, insofar as these areas showed parallel increases in Fos expression and have been implicated in the contextual process related to fear and anxiety (Walker and Davis 1997; Sullivan et al. 2004; Waddell et al. 2006).

It is interesting that several brain structures exhibited equal elevations in c-Fos expression in the EXT-SAME and EXT-DIFF conditions, and therefore exhibited c-Fos activity that was uncoupled from the expression of conditional freezing. For example, ventral CA1, ventral dentate gyrus, BL, and CEl all exhibited similar increases in c-Fos expression in the EXT-SAME and EXTDIFF condition, despite the different levels of conditional freezing among rats in these groups. It is conceivable that different networks of neurons in these areas contribute to the contextual retrieval of fear after extinction. In support of this possibility, Herry et al. (2008) have reported distinct populations of neurons in BL that respond selectively to an extinguished CS presented in either the extinction context or a renewal context. The fact that distinct populations of neurons exhibit different response patterns to an extinguished CS might account for the failure to observe correlations between c-Fos expression and conditional freezing in these brain areas.

In addition to the prefrontal cortex, amygdala, and hippocampus, we observed increased c-Fos expression in the PAGv, DRN, and NAcc shell of rats, renewing fear after extinction. Interestingly, both the medial prefrontal cortex and hippocampus send projections to the NAcc and DRN (Thierry et al. 2000), and these projections may recruit these areas during contextdependent fear renewal. The possible involvement of NAcc and DRN in the renewal of fear is consistent with the other data, indicating that the structures play a role in aversive learning and memory (Maier et al. 1993; Pezze et al. 2001; Levita et al. 2002; Thomas et al. 2002). In contrast, activity in the PAGv is most likely related to the behavioral expression of fear itself, insofar as it is known to be involved in the expression of freezing behavior (Kim et al. 1993; De Oca et al. 1998). Indeed, c-Fos expression in PAGv was similar in both the EXT-DIFF and NO-EXT conditions, which are both associated with high levels of conditional freezing. In the DRN and NAcc (as well as PRL and CEm), c-Fos expression was elevated only in the EXT-DIFF condition. This suggests that different neural circuits are engaged in the context-dependent expression of fear to an extinguished CS on the one hand, and context-independent fear to a nonextinguished CS on the other.

In contrast to the present study, Gonzalez-Lima and colleagues failed to observe increased activity in the hippocampus,

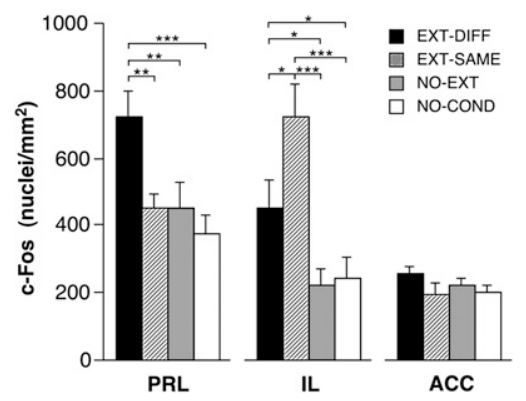

Figure 3. Mean ( \pm SEM) number of c-Fos immunopositive cell nuclei \pm SEM in the medial prefrontal cortex. (PRL) Prelimbic cortex; (IL) infralimbic cortex; (ACC) anterior cingulate cortex. $\left(^{*}\right) P<0.05 ;\left({ }^{* *}\right) P<0.01$; $\left({ }^{* * *}\right) P<0.001$. 

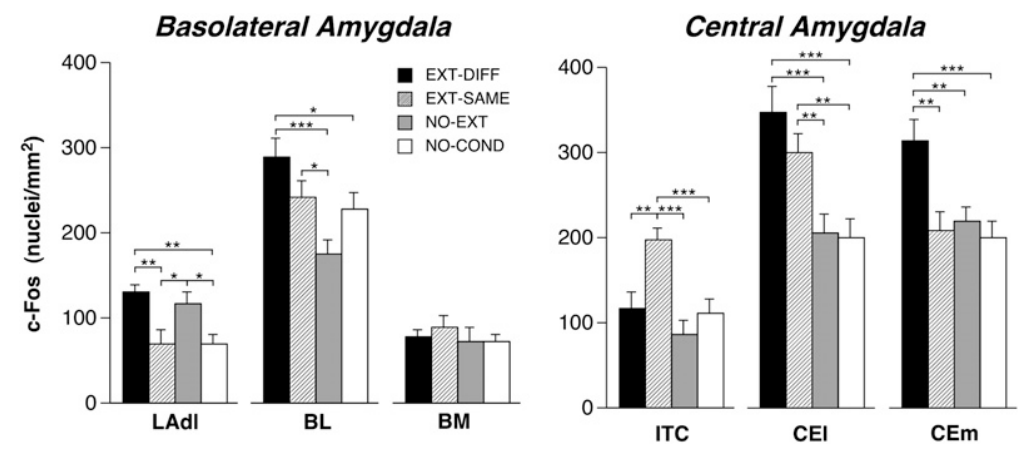

Figure 4. Mean ( \pm SEM) number of $c$-Fos immunopositive cell nuclei $\pm S E M$ in the basolateral and central amygdala. (BL) Basolateral nucleus of the amygdala; (BM) basomedial nucleus of the amygdala; (CEI) central nucleus of the amygdala, lateral division; (CEm) central nucleus of the amygdala, medial division; (LAdl) lateral nucleus of the amygdala, dorsal division; (ITC) intercalated nuclei of the amygdala. $\left({ }^{*}\right) P<0.05 ;\left({ }^{* *}\right) P<0.01 ;\left({ }^{* *}\right) P<0.001$.

amygdala, and prefrontal cortex after the renewal of fear using metabolic mapping techniques (Bruchey and Gonzalez-Lima 2006; Bruchey et al. 2007). They found that renewal was associated with increased metabolic activity in auditory and somatosensory brain areas relative to rats that received unpaired CS and US presentations during conditioning and exposure to the CS during the extinction phase of the behavioral procedure. Unfortunately, the interpretation of these data is complicated by the fact that the authors omitted two essential control groups: (1) a group that was conditioned, extinguished, and tested in the extinction context, and (2) a group that was conditioned but not extinguished. As a consequence, it is not clear whether the metabolic changes they report in their renewal condition were due to conditioning, extinction, or renewal. Moreover, rats in their renewal condition received several extinction trials in the context later used for the renewal test, a procedure that would diminish renewal and reduce the context specificity of extinction.

In conclusion, our results suggest that the context specificity of extinction is mediated by prefrontal regulation of amygdala activity, and that the hippocampus may have a fundamental role in this process. Specifically, our results reveal that the retrieval of fear and extinction memories engage reciprocal circuits in the medial prefrontal cortex and amygdala involved in the expression and suppression of fear. We suggest that contextual stimuli recruit hippocampal circuitry to regulate where and when fear is expressed. The contextual regulation of fear expression may be mediated by direct projections from the hippocampus to either (or both) the medial prefrontal cortex and/or amygdala. In the extinction context, activity in the IL and ITC suppresses fear by limiting amygdala output, whereas during the renewal of fear, activity in PRL and BL drives fear expression by increasing amygdala output. Identifying the large-scale neural networks involved in regulating fear memory after extinction is essential for understanding how dysfunction in these circuits might contribute to disorders of fear and anxiety, such as post-traumatic stress disorder.

\section{Materials and Methods}

\section{Subjects}

The subjects were 32 adult male Long-Evans rats (200-224 g) obtained from a commercial supplier (Harlan Sprague-Dawley). The rats were individually housed in clear plastic cages hanging from a standard stainless-steel rack. All animals were kept under a 14/10 light-dark cycle, with food and water provided ad libitum. The rats were handled for $5 \mathrm{~d}$ to habituate them to the experimenter.

\section{Apparatus}

Eight identical observation chambers $(30 \times 24 \times 21 \mathrm{~cm}$; MED-Associates $)$ were used for all phases of the experiment. The chambers were constructed from aluminum (two side walls) and Plexiglas (rear wall, ceiling, and hinged front door) and were situated in sound-attenuating chests located in an isolated room. The floor of each chamber consisted of 19 stainless-steel rods (4-mm diameter) spaced $1.5-\mathrm{cm}$ apart. The rods were wired to a shock source and solid-state grid scrambler (MED-Associates) for delivery of the footshock US. A speaker mounted outside of a grating in one wall of the chamber was used for the delivery of acoustic CS. Sensory stimuli were adjusted within these chambers to generate two distinct contexts (A and $\mathrm{B}$ ). For context A, a 15-W house light mounted opposite the speaker was turned on, and the fluorescent room lights remained on. The chambers were cleaned with a $1 \%$ acetic acid solution, and stainless-steel pans containing a thin film of the same solution were placed underneath the grid floors before the rats were placed inside to provide a distinct odor. Ventilation fans in each chest supplied background noise (65 dB). Rats were transported to this context in white plastic boxes. For context B, all room and chamber house lights were turned off; a pair of $40 \mathrm{~W}$ red lights provided illumination. Additionally, the doors on the sound attenuating cabinets were closed, the ventilation fans were turned off, and the chambers were cleaned with a $1 \%$ ammonium hydroxide solution. To provide a distinct odor, stainless-steel pans containing a thin film of this solution were placed underneath the grid floors before the rats were placed inside. Rats were transported to this context in black plastic boxes.

\section{Behavioral procedure}

Rats ( $n=8$ per group) were submitted to three phases of training as follows: fear conditioning, extinction, and retrieval testing. For fear conditioning, rats were transported in squads of eight and placed in the conditioning chambers in context A. The rats received five tone (10 sec; $80 \mathrm{~dB} ; 2 \mathrm{kHz}$ )-footshock (1 sec; $1 \mathrm{~mA}$ ) trials (60-sec intertrial interval [ITI]) beginning 3 min after being placed in the chambers. Sixty seconds after the final shock, the rats were returned to their home cages. Twenty-four hours after the conditioning session, rats were assigned to two groups that were extinguished to the tone either in the training context (context $\mathrm{A}$, EXT-DIFF group) or in a novel context (context B, EXT-SAME group). On the extinction day, each rat spent $55 \mathrm{~min}$ in both context $\mathrm{A}$ and context $\mathrm{B}$. In the extinction context, rats received 45 tone CS presentations (10 sec; $80 \mathrm{~dB} ; 2 \mathrm{kHz} ; 60 \mathrm{sec}$ ITI) 2 min after placement in the context, whereas in the other context, rats

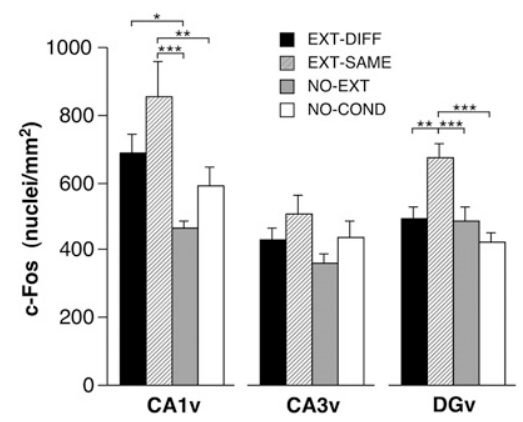

Figure 5. Mean ( \pm SEM) number of c-Fos immunopositive cell nuclei \pm SEM in the hippocampal formation. (CA1v) ventral CA1; (CA3v) ventral CA3; (DGv) ventral dentate gyrus. $\left({ }^{*}\right) P<0.05 ;\left({ }^{* *}\right) P<0.01 ;\left({ }^{* * *}\right) P<$ 0.001 . 

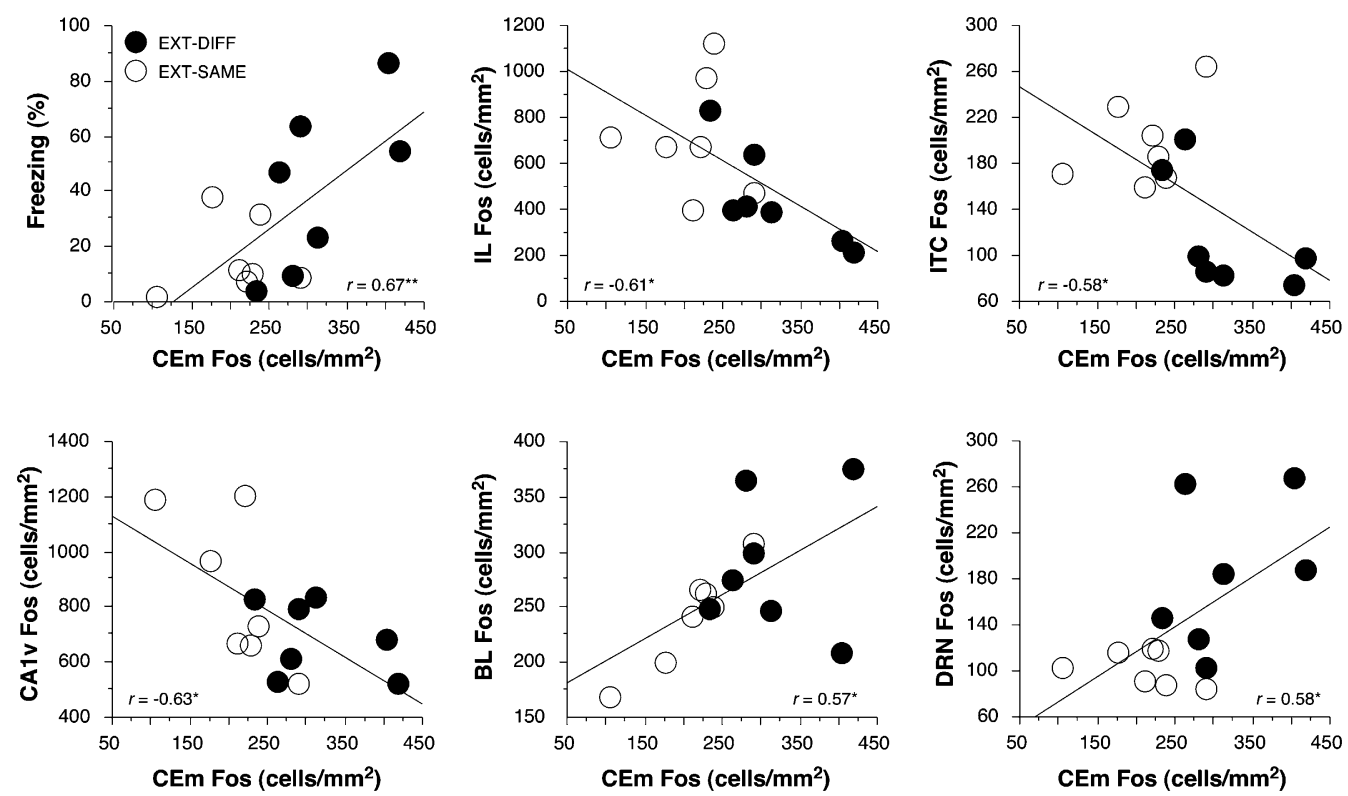

Figure 6. Significant correlations between c-Fos expression in CEm and conditional freezing (top, left) and c-Fos expression in other brain regions. $\left(^{*}\right)$ $P<0.05 ;(* *) P<0.01$.

received no tone presentations. The retrieval testing phase took place $24 \mathrm{~h}$ after the extinction session in context B for all rats. Testing consisted of two 10 -sec tone CS presentations $(80 \mathrm{~dB} ; 2$ $\mathrm{kHz}$; 60 -sec ITI) beginning 2 min after placement in the context. We delivered only two test trials to reduce the amount of extinction learning associated with the test procedure. We included control groups that either received exposure to the auditory stimuli but were not conditioned (NO-COND group) or were conditioned and not extinguished (NO-EXT group). Each control group received the same exposure to the contexts as the groups undergoing extinction training.

Fear to the tone CS during the extinction and testing phases was assessed by measuring freezing behavior. Each conditioning chamber rested on a load-cell platform that was used to record chamber displacement in response to each rat's motor activity. To ensure interchamber reliability, we calibrated each load-cell amplifier to a fixed chamber displacement. The output of each chamber's load cell was set to a gain that was optimized for detecting freezing behavior. Load-cell amplifier output from each chamber was digitized and acquired online using Threshold Activity software (MED-Associates). Absolute values of the loadcell voltages were computed and multiplied by 10 to yield a loadcell activity scale that ranged from 0 to 100 . For each chamber, load-cell activity was digitized at $5 \mathrm{~Hz}$, yielding one observation per rat every $200 \mathrm{msec}$ (300 observations/rat/minute). In all experiments, freezing was quantified by computing the number of observations for each rat that had a value less than the freezing threshold (load-cell activity $=10$; when animals exhibit freezing, load-cell activity is at or below this value). To avoid counting momentary inactivity as freezing, we scored an observation as freezing only if it fell within a contiguous group of at least five observations that were all less than the freezing threshold. Thus, freezing was only scored if the rat was immobile for at least $1 \mathrm{sec}$. For each session, the freezing observations were transformed to a percentage of total observations.

\section{c-Fos immunocytochemistry}

Ninety minutes after the onset of the first conditioned stimulus during the retrieval test session, rats were anesthetized with an overdose of sodium pentobarbital and perfused intracardially with ice-cold saline followed by $4 \%$ paraformaldehyde in $0.1 \mathrm{M}$ phosphate buffer ( $\mathrm{pH}$ 7.4). The brains were removed and stored in the same fixative for $24 \mathrm{~h}$ at $4^{\circ} \mathrm{C}$, and subsequently immersed in $30 \%$ sucrose at $4^{\circ} \mathrm{C}$. The brains were then slowly and gradually frozen and sectioned at $40 \mu \mathrm{m}$ on a cryostat. The coronal brain sections containing the structures listed in Table 2 were collected (Paxinos and Watson 2007).

The immunocytochemical staining was performed on freefloating sections. The sections were washed three times in phosphate-buffered saline ( $\mathrm{pH} 7.4$ ), incubated for $10 \mathrm{~min}$ in $0.03 \%$ $\mathrm{H}_{2} \mathrm{O}_{2}$ in PBS, washed twice in PBS, and incubated with a polyclonal antibody (anti-c-Fos, 1:1000; Santa Cruz Biotechnology no. sc-52) in PBS and normal goat serum (3\%; Vector) for $48 \mathrm{~h}$ at $4^{\circ} \mathrm{C}$. The sections were then washed three times in PBS with $0.3 \%$ Triton X-100 (Sigma), incubated with goat anti-rabbit biotinylated secondary antibody (1:500; Vector) in PBS/Triton and normal goat serum (3\%) for $2 \mathrm{~h}$ at room temperature, washed three times in PBS/Triton, incubated with avidin-biotin complex (1:1000 in PBS/ Triton; Vector $\mathrm{ABC}$ kit) for $1 \mathrm{~h}$ at room temperature, and washed three times in PBS. The immunostaining reaction was developed using the oxidase-diaminobenzidine-nickel method. The sections were incubated in distilled water with diaminobenzidine (DAB; Sigma), $0.5 \mathrm{M}$ nickel chloride, and peroxidase (Sigma) for $5 \mathrm{~min}$. The staining reaction was stopped by three washes with PBS. The reaction resulted in a dark-brown stain within the nuclei of c-Fos immunoreactive neurons. The sections were mounted on slides, air dried, dehydrated in ethanol solutions and xylene, and cover slipped with Permount (Fisher Chemicals). The measure of c-Fos immunopositivity was expressed as density, determined in the following manner: For each brain section, the number of c-Fos immunopositive nuclei in a given brain structure was counted and divided by the area occupied by this structure (in millimeters squared). For hippocampal cell fields, the region of interest included the entire cell field in a particular section, and for cortical areas included the entire depth of the cortical field in a particular section. The borders of the hippocampal cell fields, cortical areas, and subcortical nuclei were determined with the use of the thionin-stained adjacent section (see Fig. 3). Image analysis was done with the aid of an image analysis computer program (Image J) on two sections per animal brains.

\section{Statistical analysis}

For each conditioning session, the freezing data were transformed to a percentage of observations. The data were analyzed with 
Table 2. Brain regions in which c-Fos expression was analyzed

\begin{tabular}{|c|c|c|c|}
\hline Brain region & Structure & Abbreviation & Bregma $(\mathrm{mm})$ \\
\hline \multirow[t]{2}{*}{ Medial prefrontal cortex } & Prelimbic cortex & PRL & 3.24 \\
\hline & Infralimbic cortex & IL & \\
\hline \multirow[t]{2}{*}{ Nucleus accumbens } & Core & NAcc core & 2.04 \\
\hline & Shell & NAcc shell & \\
\hline Cingulate cortex & Anterior cingulate cortex & $\mathrm{ACC}$ & 2.04 \\
\hline \multirow[t]{3}{*}{ Amygdala (anterior) } & Basolateral nucleus & $\mathrm{BL}$ & -1.92 \\
\hline & Central nucleus, lateral and capsular divisions & CEI & \\
\hline & Central nucleus, medial division & CEm & \\
\hline \multirow[t]{7}{*}{ Amygdala (posterior) } & Lateral nucleus, dorsolateral & LAdl & -3.00 \\
\hline & Lateral nucleus, ventrolateral & LAvl & \\
\hline & Basomedial nucleus & BM & \\
\hline & Medial nucleus, posterodorsal & MEpd & \\
\hline & Medial nucleus, posteroventral & MEpv & \\
\hline & Bed nucleus of stria terminalis & BST & \\
\hline & Intercalated nuclei & ITC & \\
\hline \multirow[t]{3}{*}{ Hippocampus (dorsal) } & CA1 field & CA1d & -3.00 \\
\hline & CA3 field & CA3d & \\
\hline & Dentate gyrus & DGd & \\
\hline \multirow[t]{3}{*}{ Hippocampus (ventral) } & CA1 field & CA1v & -5.88 \\
\hline & CA3 field & CA3v & \\
\hline & Dentate gyrus & DGv & \\
\hline Subiculum & Ventral subiculum & SUBv & -5.88 \\
\hline \multirow[t]{2}{*}{ Medial geniculate nucleus } & Dorsal & MGd & -5.88 \\
\hline & Ventral & MGv & \\
\hline \multirow{3}{*}{ Periaqueductal gray } & Dorsal & PAGd & -7.32 \\
\hline & Lateral & PAGI & \\
\hline & Ventral & PAGv & \\
\hline Raphe nucleus & Dorsal raphe nuclei & DRN & -7.32 \\
\hline
\end{tabular}

analysis of variance (ANOVA). The density of c-Fos immunopositive cell nuclei was analyzed using independent one-way ANOVAs for each brain structure. Post-hoc comparisons in the form of Fisher's PLSD tests were performed after a significant overall $F$ ratio. All data are represented as mean \pm SEM. One rat in the EXT-SAME condition was excluded from the analysis due to a poor perfusion, and a rat in the EXT-DIFF condition was excluded as a statistical outlier.

\section{Acknowledgments}

This work was supported by a grant from the National Institutes of Health (R01MH065961 to S.M.) and the Foundation for Polish Science (KOLUMB fellowship to E.K.).

\section{References}

Akirav I, Raizel H, Maroun M. 2006. Enhancement of conditioned fear extinction by infusion of the $\mathrm{GABA}_{\mathrm{A}}$ agonist muscimol into the rat prefrontal cortex and amygdala. Eur J Neurosci 23: 758-764.

Berretta S, Pantazopoulos H, Caldera M, Pantazopoulos P, Paré D. 2005. Infralimbic cortex activation increases c-Fos expression in intercalated neurons of the amygdala. Neuroscience 132: 943-953.

Bouton ME, Mineka S, Barlow DH. 2001. A modern learning theory perspective on the etiology of panic disorder. Psychol Rev 108: 4-32.

Bouton ME, García-Gutiérrez A, Zilski J, Moody EW. 2006. Extinction in multiple contexts does not necessarily make extinction less vulnerable to relapse. Behav Res Ther 44: 983-994.

Bruchey AK, Gonzalez-Lima F. 2006. Brain activity associated with fear renewal. Eur J Neurosci 24: 3567-3577.

Bruchey AK, Shumake J, Gonzalez-Lima F. 2007. Network model of fear extinction and renewal functional pathways. Neuroscience 145: 423437.

Corcoran KA, Maren S. 2001. Hippocampal inactivation disrupts contextual retrieval of fear memory after extinction. J Neurosci 21: 1720-1726.

Corcoran KA, Maren S. 2004. Factors regulating the effects of hippocampal inactivation on renewal of conditional fear after extinction. Learn Mem 11: $598-603$.

Corcoran KA, Quirk GJ. 2007. Activity in prelimbic cortex is necessary for the expression of learned, but not innate, fears. J Neurosci 27: 840-844.

Corcoran KA, Desmond TJ, Frey KA, Maren S. 2005. Hippocampal inactivation disrupts the acquisition and contextual encoding of fear extinction. J Neurosci 25: 8978-8987.
De Oca BM, DeCola JP, Maren S, Fanselow MS. 1998. Distinct regions of the periaqueductal gray are involved in the acquisition and expression of defensive responses. J Neurosci 18: 3426-3432.

Effting M, Kindt M. 2007. Contextual control of human fear associations in a renewal paradigm. Behav Res Ther 45: 2002-2018.

Gabbott PL, Warner TA, Jays PR, Bacon SJ. 2003. Areal and synaptic interconnectivity of prelimbic (area 32), infralimbic (area 25) and insular cortices in the rat. Brain Res 993: 59-71.

Gilmartin MR, McEchron MD. 2005. Single neurons in the medial prefrontal cortex of the rat exhibit tonic and phasic coding during trace fear conditioning. Behav Neurosci 119: 1496-1510.

Hefner K, Whittle N, Juhasz J, Norcross M, Karlsson RM, Saksida LM, Bussey TJ, Singewald N, Holmes A. 2008. Impaired fear extinction learning and cortico-amygdala circuit abnormalities in a common genetic mouse strain. J Neurosci 28: 8074-8085.

Hermans D, Craske MG, Mineka S, Lovibond PF. 2006. Extinction in human fear conditioning. Biol Psychiatry 60: 361-368.

Herry C, Mons N. 2004. Resistance to extinction is associated with impaired immediate early gene induction in medial prefrontal cortex and amygdala. Eur J Neurosci 20: 781-790.

Herry C, Ciocchi S, Senn V, Demmou L, Müller C, Lüthi A. 2008. Switching on and off fear by distinct neuronal circuits. Nature 454: 600-606.

Hobin JA, Goosens KA, Maren S. 2003. Context-dependent neuronal activity in the lateral amygdala represents fear memories after extinction. J Neurosci 23: 8410-8416.

Hobin JA, Ji J, Maren S. 2006. Ventral hippocampal muscimol disrupts context-specific fear memory retrieval after extinction in rats. Hippocampus 16: 174-182.

Ji J, Maren S. 2005. Electrolytic lesions of the dorsal hippocampus disrupt renewal of conditional fear after extinction. Learn Mem 12: 270276.

Ji J, Maren S. 2007. Hippocampal involvement in contextual modulation of fear extinction. Hippocampus 17: 749-758.

Ji J, Maren S. 2008. Differential roles for hippocampal areas CA1 and CA3 in the contextual encoding and retrieval of extinguished fear. Learn Mem 15: $244-251$.

Kim JJ, Rison RA, Fanselow MS. 1993. Effects of amygdala, hippocampus, and periaqueductal gray lesions on short- and long-term contextual fear. Behav Neurosci 107: 1093-1098.

Knapska E, Radwanska K, Werka T, Kaczmarek L. 2007. Functional internal complexity of amygdala: Focus on gene activity mapping after behavioral training and drugs of abuse. Physiol Rev 87: 1113-1173.

Lebrón K, Milad MR, Quirk GJ. 2004. Delayed recall of fear extinction in rats with lesions of ventral medial prefrontal cortex. Learn Mem 11: 544548 . 
Levita L, Dalley JW, Robbins TW. 2002. Nucleus accumbens dopamine and learned fear revisited: A review and some new findings. Behav Brain Res 137: 115-127.

Likhtik E, Popa D, Apergis-Schoute J, Fidacaro GA, Paré D. 2008. Amygdala intercalated neurons are required for expression of fear extinction. Nature 454: 642-645.

Maier SF, Grahn RE, Kalman BA, Sutton LC, Wiertelak EP, Watkins LR. 1993. The role of the amygdala and dorsal raphe nucleus in mediating the behavioral consequences of inescapable shock. Behav Neurosci 107: 377-388.

Maren S. 2005. Building and burying fear memories in the brain. Neuroscientist 11: 89-99.

Maren S, Hobin JA. 2007. Hippocampal regulation of context-dependent neuronal activity in the lateral amygdala. Learn Mem 14: 318-324.

Maren S, Quirk GJ. 2004. Neuronal signalling of fear memory. Nat Rev Neurosci 5: 844-852.

Matus-Amat P, Higgins EA, Barrientos RM, Rudy JW. 2004. The role of the dorsal hippocampus in the acquisition and retrieval of context memory representations. J Neurosci 24: 2431-2439.

Milad MR, Quirk GJ. 2002. Neurons in medial prefrontal cortex signal memory for fear extinction. Nature 420: 70-74.

Milad MR, Vidal-Gonzalez I, Quirk GJ. 2004. Electrical stimulation of medial prefrontal cortex reduces conditioned fear in a temporally specific manner. Behav Neurosci 118: 389-394.

Muigg P, Hetzenauer A, Hauer G, Hauschild M, Gaburro S, Frank E, Landgraf $\mathrm{R}$, Singewald N. 2008. Impaired extinction of learned fear in rats selectively bred for high anxiety-evidence of altered neuronal processing in prefrontal-amygdala pathways. Eur J Neurosci 28: 2299_ 2309.

Myers KM, Ressler KJ, Davis M. 2006. Different mechanisms of fear extinction dependent on length of time since fear acquisition. Learn Mem 13: 216-223.

Paxinos G, Watson C. 2007. The rat brain in stereotaxic coordinates, 6th ed. Elsevier, Amsterdam, The Netherlands.

Pezze MA, Heidbreder CA, Feldon J, Murphy CA. 2001. Selective responding of nucleus accumbens core and shell dopamine to aversively conditioned contextual and discrete stimuli. Neuroscience 108: 91-102.

Quirk GJ, Mueller D. 2008. Neural mechanisms of extinction learning and retrieval. Neuropsychopharmacology 33: 56-72.

Quirk GJ, Russo GK, Barron JL, Lebron K. 2000. The role of ventromedial prefrontal cortex in the recovery of extinguished fear. J Neurosci 20: $6225-6231$.

Quirk GJ, Likhtik E, Pelletier JG, Paré D. 2003. Stimulation of medial prefrontal cortex decreases the responsiveness of central amygdala output neurons. J Neurosci 23: 8800-8807.
Rodriguez BI, Craske MG, Mineka S, Hladek D. 1999. Context-specificity of relapse: Effects of therapist and environmental context on return of fear. Behav Res Ther 37: 845-862.

Rudy JW, Matus-Amat P. 2005. The ventral hippocampus supports a memory representation of context and contextual fear conditioning: Implications for a unitary function of the hippocampus. Behav Neurosci 119: $154-163$.

Sanders MJ, Wiltgen BJ, Fanselow MS. 2003. The place of the hippocampus in fear conditioning. Eur J Pharmacol 463: 217-223.

Santini E, Ge H, Ren K, Pena de Ortiz S, Quirk GJ. 2004. Consolidation of fear extinction requires protein synthesis in the medial prefrontal cortex. J Neurosci 24: 5704-5710.

Sierra-Mercado D Jr, Corcoran KA, Lebrón-Milad K, Quirk GJ. 2006. Inactivation of the ventromedial prefrontal cortex reduces expression of conditioned fear and impairs subsequent recall of extinction. Eur $J$ Neurosci 24: 1751-1758.

Sullivan GM, Apergis J, Bush DE, Johnson LR, Hou M, LeDoux JE. 2004. Lesions in the bed nucleus of the stria terminalis disrupt corticosterone and freezing responses elicited by a contextual but not by a specific cueconditioned fear stimulus. Neuroscience 128: 7-14.

Thierry AM, Gioanni Y, Dégénétais E, Glowinski J. 2000. Hippocampoprefrontal cortex pathway: Anatomical and electrophysiological characteristics. Hippocampus 10: 411-419.

Thomas KL, Hall J, Everitt BJ. 2002. Cellular imaging with zif268 expression in the rat nucleus accumbens and frontal cortex further dissociates the neural pathways activated following the retrieval of contextual and cued fear memory. Eur J Neurosci 16: 1789-1796.

Vertes RP. 2004. Differential projections of the infralimbic and prelimbic cortex in the rat. Synapse 51: 32-58.

Vidal-Gonzalez I, Vidal-Gonzalez B, Rauch SL, Quirk GJ. 2006. Microstimulation reveals opposing influences of prelimbic and infralimbic cortex on the expression of conditioned fear. Learn Mem 13: 728-733.

Waddell J, Morris RW, Bouton M. 2006. Effects of bed nucleus of the stria terminalis lesions on conditioned anxiety: Aversive conditioning with long-duration conditional stimuli and reinstatement of extinguished fear. Behav Neurosci 120: 324-336.

Walker DL, Davis M. 1997. Double-dissociation between the involvement of the bed nucleus of the stria terminalis and the central nucleus of the amygdala in startle increases produced by conditioned versus unconditioned fear. J Neurosci 17: 9375-9383.

Wiltgen BJ, Sanders MJ, Anagnostaras SG, Sage JR, Fanselow MS. 2006. Context fear learning in the absence of the hippocampus. J Neurosci 26: 5484-5491.

Received April 21, 2009; accepted in revised form June 5, 2009. 


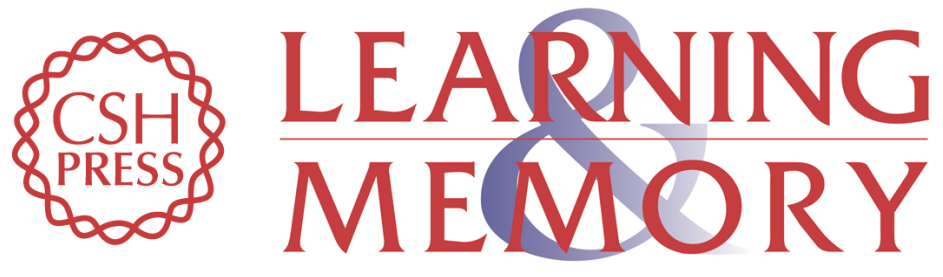

\section{Reciprocal patterns of c-Fos expression in the medial prefrontal cortex and amygdala after extinction and renewal of conditioned fear}

Ewelina Knapska and Stephen Maren

Learn. Mem. 2009, 16:

Access the most recent version at doi:10.1101/lm.1463909

References This article cites 54 articles, 19 of which can be accessed free at: http://learnmem.cshlp.org/content/16/8/486.full.html\#ref-list-1

License

Email Alerting

Receive free email alerts when new articles cite this article - sign up in the box at the Service top right corner of the article or click here. 